\title{
Zukunftswerkstatt
}

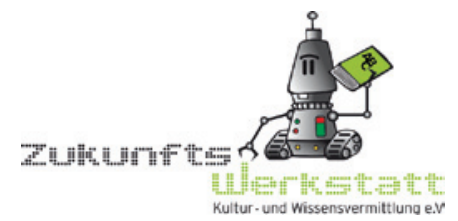

\section{„Gut arbeiten in der Zukunft“ - zur Zukunft der Arbeit}

\author{
Ein Streifzug über die Konferenzen „CoWork 2014“ und \\ „Work in Progress“
}

Die Digitalisierung verändert unser Leben und somit auch die Umstände, unter denen wir lernen und arbeiten, und das immer rasanter. Die gängige Vorstellung, dass Arbeit und Freizeit strikt voneinander zu trennen sind, „Work-Life-Balance“ also, hat sich längst in Richtung einer „Work-Life-Flexibility“ verwandelt. Und dabei stehen wir erst am Anfang der digitalen Revolution.

Wie wir auf den radikalen Wandel reagieren, den die Digitalisierung mit sich bringt, dafür müssen wir als Gesellschaft Strategien entwickeln. Wie kann sich Gesellschaft neu organisieren, wenn es immer weniger Arbeit einer bestimmten Art gibt? Wie konkret reagieren Unternehmen auf den Wandel? Inwieweit beeinflussen z. B. Coworking Spaces ${ }^{1}$ die Zukunft der Arbeit und tragen auch zu einer kreativen Stadtentwicklung bei? Und was ist das überhaupt - „gute Arbeit“?

Fragen über Fragen, die auch bei der Weiterentwicklung von Bibliotheksangeboten eine große Rolle spielen. Mit diesem Blick besuchte ich die „Cowork 2014“ vom 7. bis 9. Februar in Wolfsburg und die Konferenz „Work in Progress“, die vom 14. bis 16. März auf Kampnagel in Hamburg stattfand.

In seiner Keynote „Die Schönheit des halben Lebens“ zur „Work in Progress“ hob Professor Dr. Robert Pfaller von der Universität für angewandte Kunst in Wien hervor, dass unsere Gesellschaft schon zu über 50 Prozent aus Wissensarbeitern bestehe. Wissensarbeit wird definiert als die Bewältigung von komplexen und

\footnotetext{
1 Coworking Spaces sind Räume, die auf Tages-, Wochen- oder Monatsbasis Arbeitsplätze und Infrastruktur (Netzwerk, Drucker, Scanner, Fax, Telefon, Beamer, Besprechungsräume) zur Verfügung stellen und die Bildung einer Gemeinschaft („Community“) ermöglichen, welche mittels gemeinsamer Veranstaltungen, Workshops und weiterer Aktivitäten gestärkt werden kann. Dabei bleibt die Nutzung jedoch stets unverbindlich und zeitlich flexibel (Quelle: Wikipedia). Vgl. auch „CoWorking - ein Konzept für Bibliotheken?“, in: Bibliotheksdienst 10 (2013), S. 762-767.
} 
neuartigen Aufgaben, die sich nicht mehr mit den einmal gemachten Erfahrungen erledigen lassen. Wissensarbeiter müssen daher ständig neues Wissen erwerben, integrieren oder entwickeln. Das lasse sie insgesamt auch flexibler auf die rasanten Veränderungen der digitalen Revolution reagieren, resümierte Pfaller.

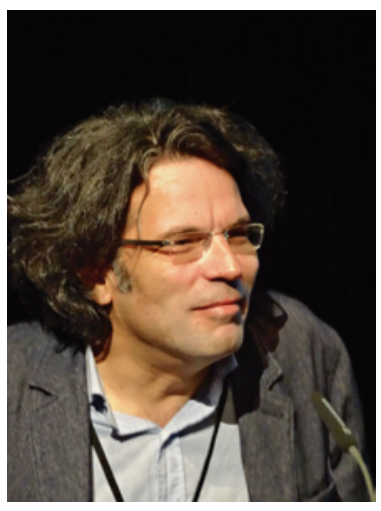

Abb. 1: Prof. Dr. Robert Pfaller (Foto: Uwe Nüstedt).

Berufsbilder und Berufungen sind also einem stetigen Wandel unterworfen. So wie wir bisher eine „gute Arbeit“ definierten - Arbeitszeit von 9 bis 17 Uhr, ein fester Arbeitsort und Kollegenkreis und das vom Berufsstart bis zur Rente -, wird es in Zukunft immer seltener der Fall sein.

Das heißt aber nicht „Leben, um zu arbeiten“ und selbstoptimiert auch die Freizeit nach dem Prinzip „höher und weiter“ effektiver gestalten müssen. Denn ob eine Arbeit eine gute Arbeit ist, hängt ganz wesentlich auch von den Verwertungsmodellen ab, die ihr zugrunde liegen, sagt Robert Pfaller.

Arbeiten wir, um mit unserem Einsatz den Produktionskreislauf eines bestimmten Produktes in Gang zu halten oder mit dem Ziel einer gewissen Perfektibilität, bei der wir den Grad der Nachhaltigkeit selbst bestimmen können? Das „Mithineinkonstruieren diverser Defekte“ in ein Produkt, damit es nach einer bestimmten Zeitspanne durch ein neues ersetzt werden muss, wie z. B. in der Automobilproduktion, ist für Pfaller ganz eindeutig kein nachhaltiges Verwertungsmodell.

Stichwort „Nachhaltigkeit“: genau an diesem Punkt setzt ja die sog. MakerBewegung (Makerspaces, FabLabs etc.) an, indem sie die „Entscheidung über die Produktionszyklen der Verantwortung des Einzelnen überlässt“. Mit den immer weiter verbreiteten 3D-Druckern bekommt mittelfristig jeder die Möglichkeit, nach seinen Wünschen und Bedürfnissen das zu produzieren, was er benötigt individuelle und kreative Produkte und keine Massenware von der Stange. Die 
Entwicklung des 3D-Druckers ist der Anfangspunkt einer neuen industriellen Revolution.

Immer mehr Kreativschaffende stellen daher für sich den Aspekt der Berufung in den Vordergrund und arbeiten an dem, was sie erschaffen wollen, ob es nun zeitgemäße Produkte oder Dienstleistungen sind. Für sie ist Arbeit eine Lebensform, in der sie mit anderen kreativen Wissensarbeitern zusammenkommen und ihre Fähigkeiten entfalten können. Zum Stichwort „gängige Vorstellung von guter Arbeit“: Wissensarbeiter und Kreativschaffende verzichten oft auf einen Arbeitsplatz innerhalb einer festen Büro- und Organisationsstruktur. Immer häufiger sind Coworking Spaces die geeigneten Orte, an denen sie ihr Lebens- und Arbeitsideal verwirklichen wollen.

Rund 50 Coworker aus ganz Deutschland und der Schweiz waren vom 7. bis 9. Februar zur deutschen Coworking-Konferenz „Cowork 2014“ nach Wolfsburg gekommen, um in Barcamp-Sessions verschiedene Aspekte des Coworkens zu thematisieren. Darunter waren Themen wie „Arbeitsstrukturen und Regeln“, die es in einem Space gibt und die oftmals so erlebten Gegensätze von kollektiven und individuellen Interessen wie produktives gemeinschaftliches versus produktives ungestörtes Arbeiten.

Wenn es im Zusammenhang mit Coworking um gruppendynamische Synergieeffekte wie Inspiration, Austausch und Vernetzung ging, hörte ich immer ganz besonders gut hin. Warum? Weil ich mir wünsche, dass das auch mehr und spontaner in Bibliotheken stattfindet: Ein gutes Beispiel dafür ist Co-Learning als Möglichkeit, sich untereinander spontan und flexibel über neue Technologien auszutauschen und Fähigkeiten dann von anderen Coworkern zu erlernen, wenn sie benötigt werden. Das, meine ich, ist gerade für Bibliotheken ein wichtiges Thema, die sich als Protagonisten im Prozess des lebenslangen Lernens neu aufstellen.

Coworking Spaces geben jungen Startups einen Raum, ihre noch unausgereiften Ideen in der Vorgründungsphase auszutesten, und betreiben damit eine Art Wirtschaftsförderung von unten. In Bezug auf eine geplante Unternehmensgründung ist das wie eine Schule zur Selbstständigkeit im Gegensatz zur Kompaktberatung, die IHKs häufig noch anbieten.

Wie werden nun Coworking Spaces und die neuen kreativen Arbeitsformen die Gesellschaft und die Zukunft der Arbeit verändern? Und welchen Einfluss haben sie auf die Entwicklung unserer Städte und ländlichen Räume? Was brauchen Städte, um kreatives Potenzial entfalten zu können?

Mit seinem Projekt „Die Stadt als Campus“ betonte in Wolfsburg Prof. Dr. Reiner Schmidt von der Hochschule Anhalt die Bedeutung neuer Arbeits-, Entfaltungs- und Experimentierräume für Bildungsprozesse in der kreativen Stadtentwicklung. Indem die Stadt zum Labor für neue urbane Lebensstile, Bildungsmo- 
delle, Arbeits- und Kooperationsformen würde, trage sie zu einer aktivierenden Stadt-, Immobilien- und Freiraumentwicklung bei.

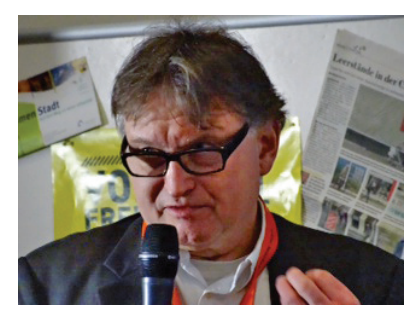

Abb. 2: Prof. Dr. Reiner Schmidt (Foto: Uwe Nüstedt).

\section{Weiterführende Informationen:}

http://cowork.mixxt.de/, http://work-in-progress-hamburg.de/ und im Blog der Zukunftswerkstatt: www.zukunftswerkstatt.org

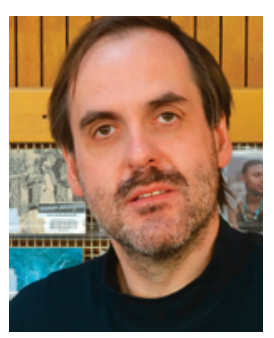

Uwe Nüstedt

Öffentlichkeitsarbeit in der Stadtbibliothek Wolfsburg und Zukunftswerkstatt 